\title{
Systematic Evaluation of the Safety and Therapeutic Effects of Para-aortic Lymphadenectomy for Advanced Gastric Cancer: a Systematic Review and Meta-analysis
}

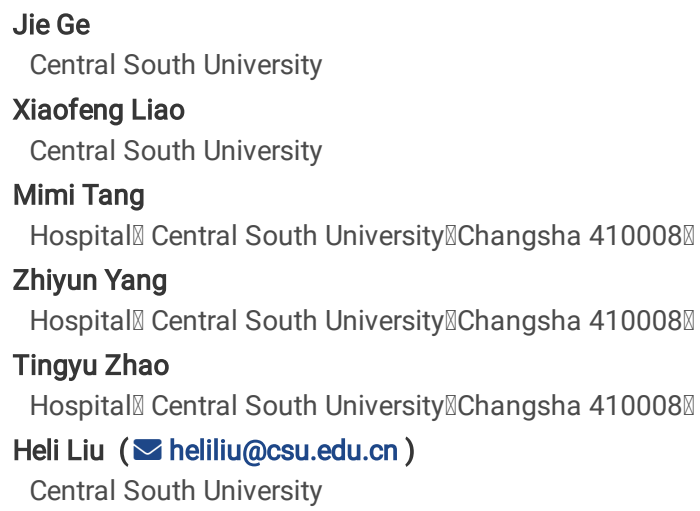




\section{Abstract}

Background: At present, there is still no definite conclusion on whether advanced gastric cancer requires additional para-aortic nodes dissection (PAND). The purpose of this study is an attempt to provide significant data to guide clinical decision-making.

Methods: The literature was searched using the terms of gastric cancer, para-aortic lymphadenectomy and D2+ lymphadenectomy and D3 lymphadenectomy. The databases searched included PubMed, Embase, Cochrane library, Web of Science, China National Knowledge Infrastructure, Wanfang Data Knowledge Service Platform, VIP Database for Chinese Technical Periodicals, and China Biology Medicine disc. RevMan 5.3 software was used for the meta-analysis.

Results: In total, 20 studies were included, consisting of 6 randomized controlled trials (RCT) and 14 non-randomized controlled trials (nRCT). These studies involved 5643 patients. The meta-analysis showed the following results: (1) compared with group D2 (D2 lymphadenectomy), the operating time in group D2+ (D2+ lymphadenectomy) was longer [mean difference (MD) $=99.45 \mathrm{~min}, 95 \%$ confidence interval $(\mathrm{Cl})(48.93,149.97), P<0.001]$, with more intra-operative blood loss [MD=262.14 mL, 95\% Cl $(165.21,359.07), P<0.001]$; (2) there were no significant differences in five-year overall survival [HR=1.09, 95\% Cl $(0.95,1.25)$, $P=0.22]$ and post-operative mortality $[R R=0.96,95 \% \mathrm{Cl}(0.59,1.57), P=0.88]$ between the two groups; (3) the rate of post-operative complication in group $\mathrm{D} 2+$ was higher than that in group $\mathrm{D} 2[\mathrm{RR}=1.42,95 \% \mathrm{Cl}(1.11,1.81), P<0.001]$

Conclusions: It is noteworthy that the combination of perioperative chemotherapy and D2+ surgery, particularly D2+ PAND, shows some survival advantages for specific patients. Although prophylactic D2+ surgery is not recommended as it increases postoperative complications and does not improve long-term survival in patients with advanced gastric cancer.

\section{Introduction}

Gastric cancer has a high morbidity and mortality, and has threatened human health worldwide for a long time. Its morbidity is ranked fifth and mortality is ranked third of all cancers ${ }^{[1-3]}$, and morbidity is highest in east Asia and lowest in North America ${ }^{[4]}$. Although early diagnosis of gastric cancer has greatly improved due to the rapid development of endoscopy, the prognosis of gastric cancer is still unsatisfactory ${ }^{[5]}$. At present, surgery is still the main therapeutic option for gastric cancer ${ }^{[6]}$. As lymph node metastasis is likely to occur in gastric cancer patients, lymph node dissection is recommended during surgery. However, the optimal extent of lymphadenectomy remains unclear and controversial.

For D1 surgery, the stomach with the primary tumor and perigastric (N1) lymph nodes are removed. Introduced by Japanese surgeons, D2 surgery is now the recommended standard practice, in which the nodes along the common hepatic, the splenic, the left gastric, and the left hepatic arteries are also removed, as well as some stations that differ for middle, proximal, and distal tumors (N2 nodes). During the late 1900s, 18\%-40\% patients with advanced GC were reported to have metastasis in the para-aortic nodes ${ }^{[7-9]}$, hence some researchers assumed that removing these lymph nodes might improve the clinical outcome of advanced $\mathrm{GC}^{[7,10,11]}$. As a consequence, D2+ surgery, the N1, N2, and the lymph nodes of other groups were further dissected, for example station 10 or station $14 \mathrm{v}$ has been developed ${ }^{[12]}$. Para-aortic lymph node is considered as the outmost barrier before gastric cancer cells systemic metastasis, so the No. 16 lymph nodes have been a focus of attention in gastrointestinal surgeons for a long time. However, there is still dispute regarding the scope of lymph node dissection.

In 1999, two studies were published in Europe ${ }^{[13,14]}$, which showed that compared with D1 surgery, not only was there no increase in the long-term survival rate following D2 surgery, but post-operative complications and mortality were higher. In 2006, a randomized controlled trial (RCT) showed that with sufficient and modern medical technology, D2 surgery had obvious survival advantages over D1 surgery ${ }^{[15]}$. At present, a consensus has been reached on D2 surgery; however, there is still no definite conclusion on whether advanced gastric cancer requires additional para-aortic lymphadenectomy (D2+ surgery). Although a systematic review published in 2010 draw a conclusion that D2 + PAND can be performed as safely as standard D2 resection without increasing postoperative mortality, but D2 + PAND fail to benefit overall survival in patients with advanced gastric cancer ${ }^{[16]}$. Simultaneously, numerous studies on D2+ surgery has been published in the past 10 years, thus the present study is dedicated to systematically evaluate relevant clinical studies comparing D2+ and D2 surgery in an attempt to provide significant data to aid clinical decision-making.

\section{Methods}

\subsection{Search strategy}

Relevant articles were retrieved from PubMed, Embase, Cochrane library, Web of Science, China National Knowledge Infrastructure (CNKI), Wanfang Data Knowledge Service Platform, VIP Database for Chinese Technical Periodicals, and China Biology Medicine disc databases. The deadline for retrieval was July, 2021.

English search terms were gastric cancer, gastric carcinoma, carcinoma of stomach, stomach cancer, para-aortic lymphadenectomy, D2+ lymphadenectomy, D2 lymphadenectomy and D2 plus, and articles were retrieved with subject terms and key words. Moreover, the references in review articles were read and included in relevant clinical controlled studies. This study was approved by the Ethic Committee of the Xiangya Hospital of Central South University (N0.201903123). The informed consent was waived by the ethics committee, since the data from previous clinical trials in which informed consent has already been obtained by the trial investigators.

\subsection{Selection of articles}


The following inclusion criteria were used: (1) Research type: RCT and nRCT; (2) Research subject: curable patients with gastric cancer, with no limit regarding age or sex; (3) Intervention measure: The control group underwent D2 surgery, while the treatment group underwent D2+ (para-aortic lymphadenectomy) surgery; (4) Evaluation indicators: operation time, intra-operative blood loss, post-operative complications, 5-year overall survival (OS), post-operative mortality and intra-operative blood transfusion.

Exclusion criteria were as follows: (1) Non-human study; (2) Patients with metastatic gastric cancer; (3) D1 surgery.

\subsection{Literature evaluation}

The Newcastle-Ottawa Scale (NOS) was used for quality evaluation of non-randomized controlled trials (nRCTs), and the Cochrane bias risk evaluation tool was used for quality evaluation of RCTs.

\subsection{Data extraction}

Two researchers independently evaluated all retrieved studies, read the titles and abstracts, selected potentially useful studies according to the inclusion and exclusion criteria, read the text thoroughly and decided which studies should be included in the meta-analysis. If there were several articles related to one study, the article containing the most complete data was included. In case of dispute on selected studies between the researchers, it was resolved by discussion.

\subsection{Statistical analysis}

RevMan 5.3 software (Cochrane Collaborative software) was used to process the data ${ }^{[17][11]}$. The HR or RR value and $95 \% \mathrm{Cl}$ were adopted for dichotomous variables to conduct a statistical analysis of the corresponding indicators. The mean difference (MD) and $95 \% \mathrm{Cl}$ were adopted for continuous variables to conduct a statistical analysis of the corresponding indicators. $P<0.05$ was considered statistically significant. The $Q$ statistics test and $\mathrm{I}^{2}$ test were used to evaluate heterogeneity. If $P>0.1$ and $\mathrm{I}^{2}<50 \%$, no heterogeneity was present. We assessed clinical heterogeneity by examining the type of participant, interventions and outcomes in each study. We assessed statistical heterogeneity using the $\mathrm{Chi}^{2}$ test to determine whether the observed differences in results were compatible with chance alone, and using the $\mathrm{I}^{2}$ statistic to determine the impact of heterogeneity on the meta-analysis. When the heterogeneity was large, the random-effects model was used. Otherwise, the fixed-effects model was used.

\section{Results}

\subsection{Article retrieval and selection}

The process of literature searching and study inclusion was shown in Figure 1. In total, 2324 articles were retrieved in databases, after delete repeated and widely different articles, 248 articles were retrieved. After reading the titles of the abstract, only 42 remained. After reading the articles and excluding articles that did not meet the inclusion criteria, 16 articles were finally selected. In addition, after reading the references of relevant review articles, 4 articles on related clinical controlled research were selected. Thus, 20 articles were selected in total involving 5643 patients.

\subsection{General characteristics and quality evaluation of the articles}

The general characteristics of all articles are shown in Table $1^{[17-29]}$. Quality evaluation of the articles was conducted by two researchers independently as it is subjective in nature. A third researcher evaluated the disputed results and resolved the disputes. 
Table 1

General characteristics of the selected articles

\begin{tabular}{|c|c|c|c|c|c|c|c|c|c|}
\hline Author & Year & Research type & Country & D2 & D2+ & D2 & D3 & D2(year) & D2+(year) \\
\hline & & & & cases & cases & male/female & male/female & age & age \\
\hline Maeta ${ }^{[17]}$ & 1999 & RCT & Japan & 35 & 35 & $21 / 14$ & $20 / 15$ & $60 \pm 11$ & $59 \pm 9$ \\
\hline Ikeguchi $[40]$ & 2000 & Non-RCT & Japan & 524 & 221 & NA & NA & NA & NA \\
\hline Jiang $^{[18]}$ & 2000 & RCT & China & 32 & 21 & $19 / 13$ & $11 / 10$ & $46-83$ & $34-84$ \\
\hline Yan-Chang ${ }^{[19]}$ & 2002 & Non-RCT & China & 50 & 36 & NA & $22 / 14$ & $29-70$ & $34-68$ \\
\hline Bostanci[20] & 2004 & Non-RCT & Turkey & 100 & 34 & $63 / 37$ & $21 / 13$ & $58.5 \pm 13$ & $53 \pm 12.6$ \\
\hline Kunisaki $^{[21]}$ & 2006 & Non-RCT & Japan & 430 & 150 & $286 / 144$ & $109 / 41$ & $62.2 \pm 12.5$ & $59.3 \pm 10.7$ \\
\hline Yonemura $^{[22]}$ & 2006 & RCT & Japan & 128 & 128 & $84 / 44$ & $86 / 42$ & $63.8 \pm 9.7$ & $62.5 \pm 10.2$ \\
\hline Zhan [41] & 2006 & Non-RCT & China & 146 & 126 & $92 / 34$ & $113 / 33$ & NA & NA \\
\hline Kulig [23] & 2007 & RCT & Poland & 141 & 134 & $85 / 56$ & $83 / 51$ & $31-81$ & $34-77$ \\
\hline Sasako [25] & 2008 & RCT & Japan & 263 & 260 & $176 / 87$ & $183 / 77$ & $25-75$ & $25-75$ \\
\hline Yonemura ${ }^{[24]}$ & 2008 & $\mathrm{RCT}$ & Japan & 135 & 134 & $90 / 45$ & $91 / 43$ & $63.8 \pm 9.7$ & $62.5 \pm 10.2$ \\
\hline $\mathrm{Hu}{ }^{[27]}$ & 2009 & Non-RCT & China & 55 & 62 & $42 / 13$ & $48 / 14$ & $58.8 \pm 11.4$ & $54.3 \pm 11.4$ \\
\hline HUANG [26] & 2009 & Non-RCT & China & 101 & 50 & $77 / 24$ & $35 / 15$ & $52.3 \pm 11.2$ & $52.6 \pm 12.5$ \\
\hline Zheng [28] & 2011 & Non-RCT & China & 33 & 42 & $19 / 14$ & $29 / 13$ & $53.5 \pm 11.2$ & $55.9 \pm 8.9$ \\
\hline Bostanci ${ }^{[29]}$ & 2013 & Non-RCT & Turkey & 370 & 98 & $248 / 122$ & $61 / 37$ & $60.2 \pm 12.4$ & $56.2 \pm 11.9$ \\
\hline liang ${ }^{[42]}$ & 2013 & Non-RCT & China & 109 & 44 & $72 / 37$ & $25 / 19$ & NA & NA \\
\hline SUN [43] & 2013 & Non-RCT & China & 50 & 50 & $34 / 16$ & $35 / 15$ & $50.3 \pm 7.4$ & $50.6 \pm 8.5$ \\
\hline ZHANG ${ }^{[44]}$ & 2014 & Non-RCT & China & 88 & 69 & NA & NA & NA & NA \\
\hline zhang [45] & 2015 & Non-RCT & China & 287 & 129 & NA & NA & NA & NA \\
\hline Marrelli ${ }^{[46]}$ & 2017 & Non-RCT & Italy & 353 & 390 & NA & NA & NA & NA \\
\hline
\end{tabular}

RCTs adopt the Cochrane bias risk evaluation tool, which is edited by the Cochrane Collaborative network and is the first choice recommended by statisticians and system reviewers for evaluating $\mathrm{RCT}^{[30]}$. The evaluation results are shown in Figures 2 and 3 . According to the bias risk charts, the following conclusions were obtained: (1) For random sequence generation (selection bias), $66.7 \%$ of RCTs had low risk and risk was unclear in $33.3 \%$ of RCTs. (2) For allocation concealment (selection bias), $33.3 \%$ of RCTs had low risk, risk was unclear in $50 \%$ of RCTs, and $16.7 \%$ of RCTs had high risk. (3) For performance bias, $66.7 \%$ of RCTs had low risk, and risk was unclear in $33.3 \%$ of RCTs. (4) For detection bias, $50 \%$ of RCTs had low risk and risk was unclear in $50 \%$ of RCTs. (5) For attrition bias, $100 \%$ of RCTs had low risk. (6) For reporting bias, $83.3 \%$ of RCTs had low risk and risk was unclear in $16.7 \%$ of RCTs. (7) For unclear risk of bias, the risk of other bias was unclear in $100 \%$ of RCTs.

nRCTs adopt the NOS scale for evaluation, and the higher the score, the better the quality ${ }^{[31]}$. The total score is 9 . Specific rating is shown in Table 2. 
Table 2

Newcastle-Ottawa Scale for assessing the quality of nRCTs

\begin{tabular}{|c|c|c|c|c|c|c|c|c|c|}
\hline \multirow[t]{2}{*}{ Study ID } & & \multicolumn{4}{|l|}{ Selection } & \multicolumn{2}{|l|}{ Comparability } & \multicolumn{2}{|l|}{ Outcome } \\
\hline & & $\begin{array}{l}\text { Representativeness } \\
\text { of the exposed } \\
\text { cohort }\end{array}$ & $\begin{array}{l}\text { Selection } \\
\text { of the } \\
\text { non- } \\
\text { exposed } \\
\text { cohort }\end{array}$ & $\begin{array}{l}\text { Ascertainment } \\
\text { of exposure }\end{array}$ & $\begin{array}{l}\text { Demonstration } \\
\text { that outcome } \\
\text { of interest was } \\
\text { not present at } \\
\text { start of study }\end{array}$ & $\begin{array}{l}\text { Comparability } \\
\text { of cohorts on } \\
\text { the basis of } \\
\text { the design }\end{array}$ & $\begin{array}{l}\text { Comparability } \\
\text { of cohorts on } \\
\text { the basis of } \\
\text { the analysis }\end{array}$ & $\begin{array}{l}\text { Assessment } \\
\text { of outcome }\end{array}$ & $\begin{array}{l}\text { Was } \\
\text { follow-up } \\
\text { long } \\
\text { enough } \\
\text { for } \\
\text { outcomes } \\
\text { to occur }\end{array}$ \\
\hline $\begin{array}{l}\text { Ikeguchi } \\
{[40]}\end{array}$ & 2000 & 1 & 1 & 1 & 1 & 1 & 1 & 1 & \\
\hline $\begin{array}{l}\text { Yan- } \\
\text { Chang } \\
\text { [19] }\end{array}$ & 2002 & 1 & 1 & 1 & 1 & 1 & & & \\
\hline $\begin{array}{l}\text { Bostanci } \\
{[20]}\end{array}$ & 2004 & 1 & 1 & 1 & 1 & 1 & 1 & 1 & \\
\hline $\begin{array}{l}\text { Kunisaki } \\
\text { [21] }\end{array}$ & 2006 & 1 & 1 & 1 & 1 & 1 & 1 & 1 & 1 \\
\hline zhan [41] & 2006 & 1 & 1 & 1 & 1 & 1 & 1 & 1 & 1 \\
\hline $\mathrm{Hu}{ }^{[27]}$ & 2009 & 1 & 1 & 1 & 1 & 1 & & 1 & 1 \\
\hline $\begin{array}{l}\text { HUANG } \\
{[26]}\end{array}$ & 2009 & 1 & 1 & 1 & 1 & 1 & & & \\
\hline $\begin{array}{l}\text { Zheng } \\
\text { [28] }\end{array}$ & 2011 & 1 & 1 & 1 & 1 & 1 & & & \\
\hline $\begin{array}{l}\text { Bostanci } \\
\text { [29] }\end{array}$ & 2013 & 1 & 1 & 1 & 1 & 1 & 1 & 1 & 1 \\
\hline liang [42] & 2013 & 1 & 1 & 1 & 1 & 1 & & 1 & 1 \\
\hline SUN [43] & 2013 & 1 & 1 & 1 & 1 & 1 & & 1 & 1 \\
\hline $\begin{array}{l}\text { ZHANG } \\
\text { [44] }\end{array}$ & 2014 & 1 & 1 & 1 & 1 & 1 & & 1 & 1 \\
\hline $\begin{array}{l}\text { zhang } \\
\text { [45] }\end{array}$ & 2015 & 1 & 1 & 1 & 1 & 1 & & 1 & 1 \\
\hline $\begin{array}{l}\text { Marrelli } \\
{[46]}\end{array}$ & 2017 & 1 & 1 & 1 & 1 & 1 & & 1 & 1 \\
\hline
\end{tabular}

\subsection{Results of meta-analysis}

\section{(I) Operation time}

Data of ten studies ${ }^{[17-19,21,22,26,27]}$ were applicable. The meta-analysis results showed that MD $=99.45 \mathrm{~min}, 95 \% \mathrm{Cl}(48.93,149.97), P<0.001$, and the differences between the two groups were statistically significant. Compared with the D2 group, the operation time in the D2+ group was longer with an average of $99.45 \mathrm{~min}$ (Figure 4).

\section{(II) Intra-operative blood loss}

Five studies $^{[17,19,21,22]}$ reported intra-operative blood loss. The meta-analysis results showed that MD $=262.14 \mathrm{~mL}, 95 \% \mathrm{Cl}(165.21,359.07)$. There was a significant difference between these two groups. Blood loss in the D2+ group was significantly more than that in the D2 group, and the test for heterogeneity test was 0.03 (Figure 5).

\section{(III) Rate of post-operative complication}

Fourteen studies ${ }^{[17-23,25-29]}$ mentioned the incidence of post-operative complications. The meta-analysis results showed that the post-operative complication rate in the $\mathrm{D} 2+$ group was higher than that in the $\mathrm{D} 2$ group [RR=1.42, 95\% $\mathrm{Cl}(1.11,1.81), P<0.001]$ (Figure 6).

(IV) Post-operative mortality

Page 5/12 
Twelve studies ${ }^{[17-25,27-29]}$ mentioned post-operative mortality rate. The meta-analysis results showed that the differences between the two groups were not statistically significant. Compared with the D2 group, post-operative mortality of $\mathrm{D} 2+$ group was not increased [RR=0.96, 95\% $\mathrm{Cl}(0.59,1.57), P=0.88](\mathrm{Figure} 7)$.

\section{(V) Post-operative 5-year OS}

Seven studies ${ }^{[17,21,24,25,27,29]}$ reported post-operative 5-year OS. The meta-analysis results showed that the differences between the two groups were not statistically significant. Compared with D2 surgery, D2+ surgery would not improve post-operative OS [HR=1.09, 95\% Cl $(0.95,1.25), P=0.22](\mathrm{Figure} 8)$.

\section{Publication Bias And Sensitivity Analysis}

Funnel plots was used to make a visual assessment of publication bias, according to the Cochrane manual, Funnel plots was required when the number of studies enrolled was more than 10 in meta-analysis. the number of studies enrolled was more than 10 in operation time (figure $9 \mathrm{~A}$ ), post-operative complications (figure 9B) and post-operative mortality (figure 9C).

Sensitivity analysis was carried out by excluding each study one by one. No significant changes in sensitivity and specificity were observed.

\section{Discussion}

We conducted a systemic review based on current studies using the method provided by Cochrane Collaboration to perform a comparison of the safety and therapeutic effect between standard D2 and D2+ lymphadenectomy. Six RCTs (total 1456 patients) and fourteen non-RCTs (total 4187 patients) were included in our review. The results of meta-analysis showed that D2+ surgery will increase post-operative complication, and does not improve the long-term survival rate of patients with advanced gastric cancer.

According to the results of this meta-analysis, although there was obvious statistical heterogeneity regarding operation time, intra-operative blood loss and post-operative complication, the sensitivity and specificity did not change significantly when each study was excluded one by one. Meanwhile statistical heterogeneity regarding post-operative mortality and 5-year OS were not be observed.

There was significant difference about the rate of post-operative complication between D2 and D2+ operation, whereas high heterogeneity and sensitivity analysis resulted differently after excluding each study one after another, the reason was probably related to surgical experience. Noticeably, with modern technology as well as proficient team, D2+ surgery would be performed safely.

Compared with D2 surgery, D2+ surgery could not improve the long-term survival of patients with gastric cancer, but significant differences were found in many subgroup studies. In 2008, a RCT published by Sasako M et al found that as compared with D2 surgery, treatment with D2+ surgery does not improve the survival rate in curable gastric cancer. However, in subgroups with 5-10 cm tumor size and negative lymph node metastasis, D2+ surgery acquired better 5year OS than D2 surgery ${ }^{[25]}$. In 2009, a study by Fujimura T et al reported that patients who accepted selective removal of station 16 b1 lateral group lymph nodes and station $16 \mathrm{a} 2$ interior group lymph nodes with positive metastasis had better prognosis ${ }^{[32]}$. Similarly, a retrospective case-control conducted by Hu et al also found that the 5-year OS were $66.7 \%$ verse $56.4 \%$ in the D2+PAND and D2 groups, respectively, in those of the patients with T4 stage ${ }^{[27]}$. In 2016 , Morita $S$ et al reported D2+ surgery could not increase the long-term survival of aged patients with lymph node positive metastasis between the upper side of the left renal vein, abdominal aorta and inferior vena cava ${ }^{[33]}$. Although all studies have demonstrated D2+ surgery has certain survival advantages for specific populations, prophylactic D2+ surgery is not preferred recommendation due to evidence-based medicine remains insufficient. After all, clinicians still don't have accurate method of assessing lymph-node metastases before surgery. Multi-Detector-Row Computed Tomography (MDCT) is thought to be accurate for the diagnosis of suspected lymph node metastasis, but post-operative positive predictive value was only $73 \%$. Despite Multi-disciplinary team cooperation can improve the accuracy in lymph node metastasis evaluation, neither can provide a positive diagnosis ${ }^{[34]}$.

As well-known chemotherapy has been an effective adjuvant therapy for gastric cancer, whether combination of perioperative chemotherapy and D2+ surgery increase long-term survival? Encouraging results have been found in some studies. In 2009, Yoshikawa T et al published a study on the treatment of gastric cancer with positive 16 th lymph node by combining pre-operative chemotherapy and D2+ surgery. Although the study was terminated early due to a mortality rate in the tested population higher than $5 \%$, the results showed that preliminary multi-modal treatment improved the 3-year survival rate which was as high as $27 \%{ }^{[35]}$. In 2012, a study by Oyama K et al showed that, compared with D2+ surgery alone, D2+ surgery combined with chemotherapy could improve 2-year OS and recurrence-free survival[36]. In 2014, a study by Tsuburaya A et al showed that the 3-year and 5-year OS of patients with gastric cancer and wide-range lymph node metastasis who were treated with D2+ surgery combined with pre-operative chemotherapy reached $59 \%$ and $53 \%$, respectively ${ }^{[37]}$. In 2014 , a phase II clinical trial in Zhongshan Hospital showed compared with the simple chemotherapy group, patients receiving D2+ surgery combined with pre-operative chemotherapy had better long-term survival and progression-free survival ${ }^{[38]}$. According to these studies, combined treatment of pre-operative chemotherapy and D2+ surgery has achieved better long-term survival, but more evidence-based medicine is needed to prove the efficacy. Recent studies of JCOG 0001 and JCOG 0405 reported S-1 plus cisplatin might be preferred preoperative scheme for gastric cancer with extensive lymph node metastasis ${ }^{[39]}$. It is worth expecting that a RCT on this protocol is underway (clinical trial number: NCT02139605), which could provide more evidence for the development of clear management guidelines for lymph node dissection in gastric cancer.

\section{Conclusions}

For experienced teams, D2+ PAND surgery is feasible and safe. Although prophylactic D2+ surgery is not recommended, D2+surgery lespecially D2+PAND has some survival advantages for selected patients, and D2+ surgery to achieve R0 resection combined with chemotherapy may improve long-term survival. 
Gastric cancer patients with clinically para-aortic nodal suspected metastases, treatment model of perioperative chemotherapy combined D2+PAND surgery should be reasonable.

\section{Declarations}

\section{Acknowledgements}

We acknowledge all the authors whose publications are referred in our article.

\section{Authors' contributions}

Xiaofeng Liao collected data, designed this work and wrote the manuscript; Mimi Tang, Zhiyun Yang and Tingyu Zhao took part in the quality evaluation of the articles and article selection; Heli Liu designed this work, revised the manuscript. All authors contributed to final approval, accountability for the manuscript. The authors are grateful to doctor Songlin Zhu from Cancer Hospital of Hunan Province for his generous help on statistic analysis.

\section{Funding}

This work was partly funded by Innovation Guidance Plan (2018SK52604) of Hunan Provincial Science and Technology Department.

\section{Data availability}

The datasets used and analyzed during the current study are available from the corresponding author on reasonable request.

\section{Ethics approval and informed consent}

We obtained ethical approval from the Ethics Committee of Xiangya Hospital of Central South University(NO. 20193123). The informed consent was waived by the ethics committee mentioned above, since the data from previous clinical trials in which informed consent has already been obtained by the trial investigators. All methods were performed in accordance with the Declaration of Helsinki.

\section{Consent for publication}

All the fgures and tables in this study were original, all were made by our authors.

\section{Competing interests}

The authors declare no competing interests.

\section{References}

1. Liu, H., et al., What are the reasons for a longer operation time in robotic gastrectomy than in laparoscopic gastrectomy for stomach cancer? Surg Endosc, 2019. 33(1): p. 192-198.

2. Xiao, H., et al., Peri-Operative Blood Transfusion Does Not Influence Overall and Disease-Free Survival After Radical Gastrectomy for Stage II/III Gastric Cancer: a Propensity Score Matching Analysis. J Gastrointest Surg, 2018. 22(9): p. 1489-1500.

3. Reddavid, R., et al., Neoadjuvant chemotherapy for gastric cancer. Is it a must or a fake? World J Gastroenterol, 2018. 24(2): p. 274-289.

4. Jia, F., et al., Discordance of Somatic Mutations Between Asian and Caucasian Patient Populations with Gastric Cancer. Mol Diagn Ther, 2017. 21(2): p. 179-185.

5. Li, R., et al., Weekday of Surgery Affects Postoperative Complications and Long-Term Survival of Chinese Gastric Cancer Patients after Curative Gastrectomy. Biomed Res Int, 2017. 2017: p. 5090534.

6. Yang, X.W., et al., Outcomes of laparoscopic gastrectomy for gastric cancer in elderly patients. J buon, 2018. 23(1): p. 85-91.

7. Nakane, Y., et al., Incidence and prognosis of para-aortic lymph node metastasis in gastric cancer. Hepatogastroenterology, 1998. 45(23): p. 1901-6.

8. Isozaki, H., et al., Effectiveness of paraaortic lymph node dissection for advanced gastric cancer. Hepatogastroenterology, 1999. 46(25): p. 549-54.

9. Takashima, S. and T. Kosaka, Results and controversial issues regarding a para-aortic lymph node dissection for advanced gastric cancer. Surg Today, 2005. 35(6): p. 425-31.

10. Yonemura, Y., et al., Surgical treatment of advanced gastric cancer with metastasis in para-aortic lymph node. Int Surg, 1991. 76(4): p. 222-5.

11. Baba, M., et al., Paraaortic lymphadenectomy in patients with advanced carcinoma of the upper-third of the stomach. Hepatogastroenterology, 2000. 47(33): p. 893-6.

12. Rosa, F., et al., Classification of nodal stations in gastric cancer. Transl Gastroenterol Hepatol, 2017. 2: p. 2.

13. Cuschieri, A., et al., Patient survival after D1 and D2 resections for gastric cancer: long-term results of the MRC randomized surgical trial. Surgical Cooperative Group. Br J Cancer, 1999. 79(9-10): p. 1522-30.

14. Bonenkamp, J.J., et al., Extended lymph-node dissection for gastric cancer. N Engl J Med, 1999. 340(12): p. 908-14.

15. Wu, C.W., et al., Nodal dissection for patients with gastric cancer: a randomised controlled trial. Lancet Oncol, 2006. 7(4): p. 309-15. 
16. Wang, Z., J.Q. Chen, and Y.F. Cao, Systematic review of D2 lymphadenectomy versus D2 with para-aortic nodal dissection for advanced gastric cancer. World J Gastroenterol, 2010. 16(9): p. 1138-49.

17. Maeta, M., et al., A prospective pilot study of extended (D3) and superextended para-aortic lymphadenectomy (D4) in patients with T3 or T4 gastric cancer managed by total gastrectomy. Surgery, 1999. 125(3): p. 325-31.

18. Jiang B J, G.Y.F., Sun R X, et al., Clinical study on the dissection of lymph nodes around abdominal aortic artery in advanced gastric cancer. Chinese Journal of General Surgery, 2000. 09(04): p. 292-295.

19. Yan-Chang X U, L.J.C., Xue-Lei W U., A Study of Periaortic Lymph Nodes Dissection in Patients with Advanced Gastric Carcinoma. Zhejiang Cancer Journal, 2002. 08(05): p. 257-259.

20. Bostanci, E.B., et al., Comparison of complications after D2 and D3 dissection for gastric cancer. Eur J Surg Oncol, 2004. 30(1): p. 20-5.

21. Kunisaki, C., et al., Comparison of surgical results of D2 versus D3 gastrectomy (para-aortic lymph node dissection) for advanced gastric carcinoma: a multi-institutional study. Ann Surg Oncol, 2006. 13(5): p. 659-67.

22. Yonemura, Y., et al., Operative morbidity and mortality after D2 and D4 extended dissection for advanced gastric cancer: a prospective randomized trial conducted by Asian surgeons. Hepatogastroenterology, 2006. 53(69): p. 389-94.

23. Kulig, J., et al., Standard D2 versus extended D2 (D2+) lymphadenectomy for gastric cancer: an interim safety analysis of a multicenter, randomized, clinical trial. Am J Surg, 2007. 193(1): p. 10-5.

24. Yonemura, Y., et al., Randomized clinical trial of D2 and extended paraaortic lymphadenectomy in patients with gastric cancer. Int J Clin Oncol, 2008. 13(2): p. $132-7$.

25. Sasako, M., et al., D2 lymphadenectomy alone or with para-aortic nodal dissection for gastric cancer. N Engl J Med, 2008. 359(5): p. 453-62.

26. HUANG Yongjian, Y.J., XU Dongpo, Para-aortic Lymph Node Dissection for Gastric Cancer of Advanced Stage: A Clinical Study. China Modern Doctor, 2009. 47: p. 6-7.

27. Hu, J.K., et al., D2 plus para-aortic lymphadenectomy versus standardized D2 lymphadenectomy in gastric cancer surgery. Surg Today, 2009. 39(3): $\mathrm{p}$. 207-13.

28. Zheng J, D.F., Dian-Kui QU, et al., Clinical research of postoperative complications of D2 simple gastric carcinoma surgery and D2 gastric carcinoma surgery plus para-aortic nodal dissection. Chinese Journal of Current Advances in General Surgery, 2011. 14(12): p. $945-947$.

29. Bostanci, E.B., et al., A prospective observational study of 468 patients undergoing D2 or D3 lymphadenectomy for gastric cancer. Hepatogastroenterology, 2013. 60(123): p. 624-7.

30. 5.3), R.M.V., Cochrane Collaboration. Oxford, UK, 2014.

31. Qiong HE, H.Y., Kang WJ, et al., Quality assessment of case-control and cohort studies on injury prevention in China,2001-2010. Chinese Journal of Disease Control \& Prevention, 2014. 18(10): p. 913-916.

32. Fujimura, T., et al., Selective lymphadenectomy of para-aortic lymph nodes for advanced gastric cancer. Oncol Rep, 2009. 22(3): p. 509-14.

33. Morita, S., et al., The clinical significance of para-aortic nodal dissection for advanced gastric cancer. Eur J Surg Oncol, 2016. 42(9): p. 1448-54.

34. Wang W, Xiaojiang Y, Jin w, Clinical significance of abdominal paraaortic lymph node dissection in gastric cancer. Chinese Journal of General Surgery, 27(4): p. 391-395.

35. Yoshikawa, T., et al., Phase Il study of neoadjuvant chemotherapy and extended surgery for locally advanced gastric cancer. Br J Surg, 2009. 96(9): $\mathrm{p}$. 1015-22.

36. Oyama, K., et al., Efficacy of pre-operative chemotherapy with docetaxel, cisplatin, and S-1 (DCS therapy) and curative resection for gastric cancer with pathologically positive para-aortic lymph nodes. J Surg Oncol, 2012. 105(6): p. 535-41.

37. Tsuburaya, A., et al., Neoadjuvant chemotherapy with S-1 and cisplatin followed by D2 gastrectomy with para-aortic lymph node dissection for gastric cancer with extensive lymph node metastasis. Br J Surg, 2014. 101(6): p. 653-60.

38. Wang, Y., et al., A phase II trial of Xeloda and oxaliplatin (XELOX) neo-adjuvant chemotherapy followed by surgery for advanced gastric cancer patients with para-aortic lymph node metastasis. Cancer Chemother Pharmacol, 2014. 73(6): p. 1155-61.

39. Katayama, H., et al., An integrated analysis of two phase II trials (JCOG0001 and JCOG0405) of preoperative chemotherapy followed by D3 gastrectomy for gastric cancer with extensive lymph node metastasis. Gastric Cancer, 2019. 22(6): p. 1301-1307.

40. Ikeguchi, M., et al., Prognostic benefit of extended radical lymphadenectomy for patients with gastric cancer. Anticancer Res, 2000. 20(2b): p. 1285-9.

41. ZHAN Wen-hua $\triangle$ HAN Fang-hai囚HE Yu-long, L.Y.-m.Z.Z.-q.P.J.-s.C.S.-r., DisdpHnarian of lymph node metastasis and effect of paraaortic lymph nodes dissection on clinicaloutcomes in advanced gastric carcinoma. Chinese Journal of Gastrointestinal surgery, 2006. 9(01): p. 17-22.

42. LIANG Yue-xiang, L.H., DING Xue-wei, WANG Xiao-na, ZHANG Li, WU Liang-liang, LIU Hong-gen, JIAO Xu-guang, The prognostic influence of D2 lymphadenectomy with para-aortic lymph nodal dissection for gastric cancer in N3 stage. Chinese Journal of Surgery, 2013. 51(12): p. 1071-1076.

43. Bo, S., No. $16 a 2 b 1$ lymph node dissection for advanced gastric cancer prolongs patients'survival. Chinese Journal of General Surgery, 2013. 28(10): $\mathrm{p}$. 744-747.

44. Zhang, C., et al., Evaluation of para-aortic nodal dissection for locoregionally advanced gastric cancer with 1-3 involved para-aortic nodes. Chin Med J (Engl), 2014. 127(3): p. 435-41.

45. Zhang, C.D., et al., Prognostic significance of distal subtotal gastrectomy with standard D2 and extended D2 lymphadenectomy for locally advanced gastric cancer. Sci Rep, 2015. 5: p. 17273.

Page 8/12 
46. Marrelli, D., et al., Incidence and Prognostic Value of Metastases to "Posterior" and Para-aortic Lymph Nodes in Resectable Gastric Cancer. Ann Surg Oncol, 2017. 24(8): p. 2273-2280.

\section{Figures}

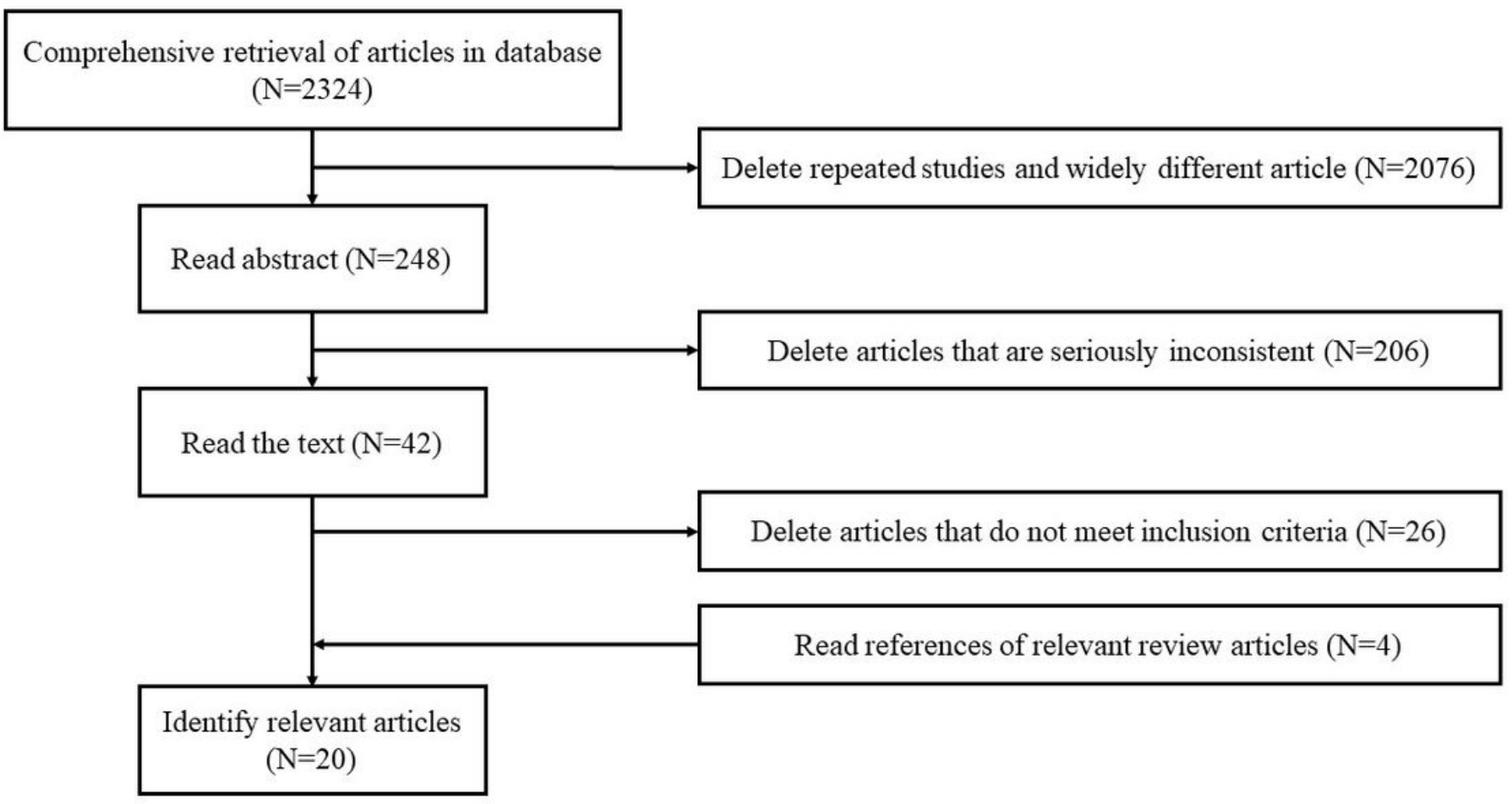

Figure 1

Legend not included with this version.

\section{Random sequence generation (selection bias)}

Allocation concealment (selection bias)

Blinding of participants and personnel (performance bias)

Blinding of outcome assessment (detection bias)

Incomplete outcome data (attrition bias)

Selective reporting (reporting bias)

Other bias
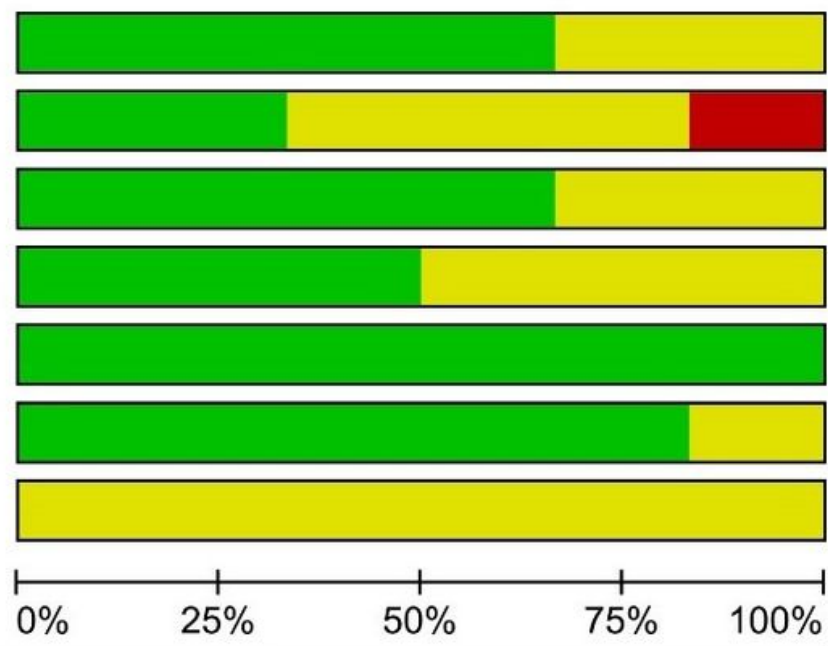

Low risk of bias
High risk of bias

Figure 2

Legend not included with this version. 


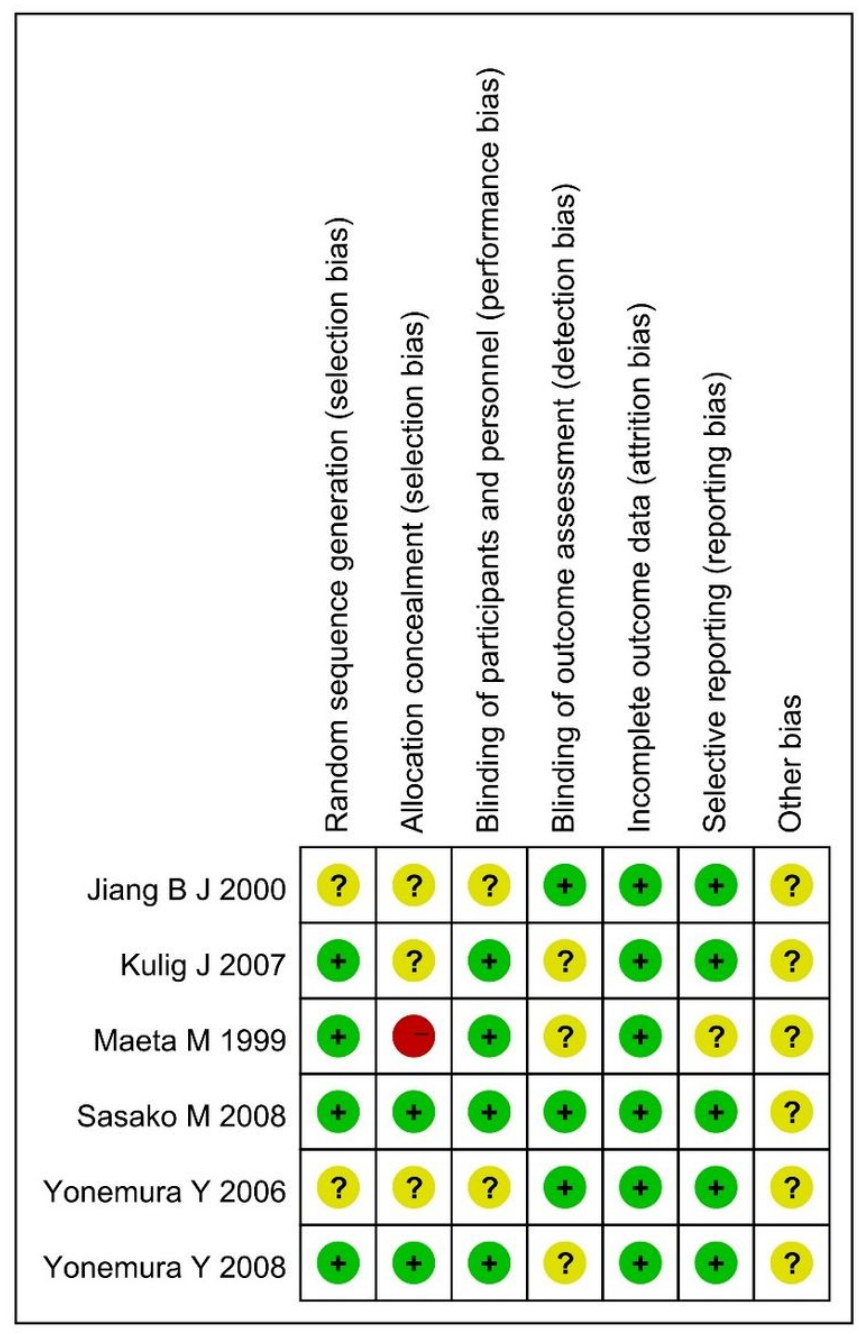

Figure 3

Legend not included with this version.

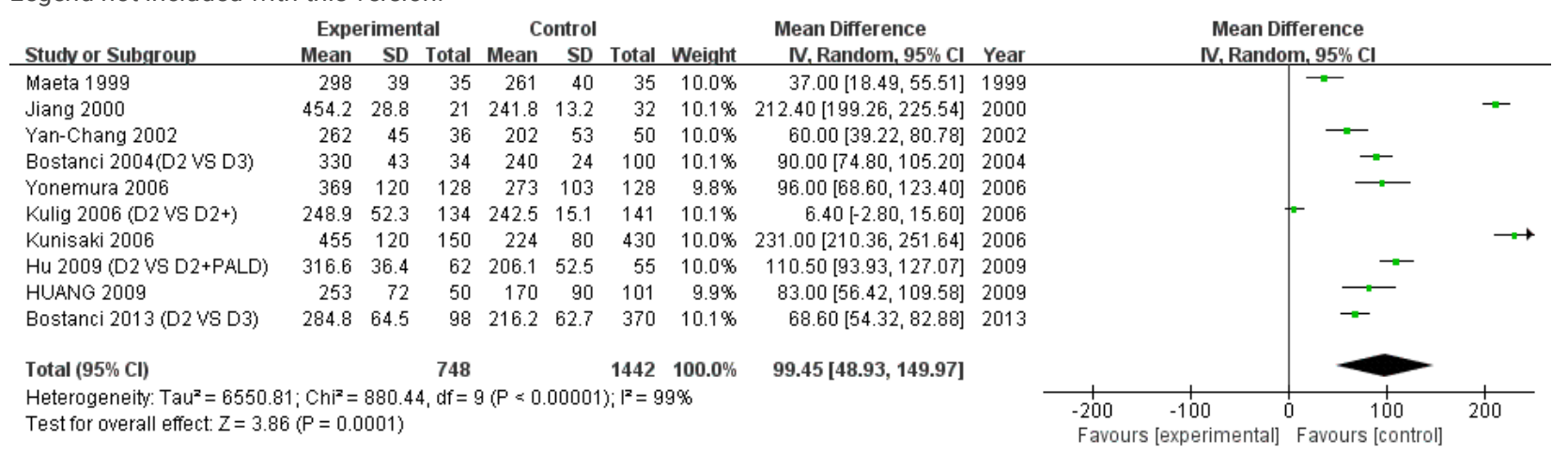

Figure 4

Legend not included with this version.

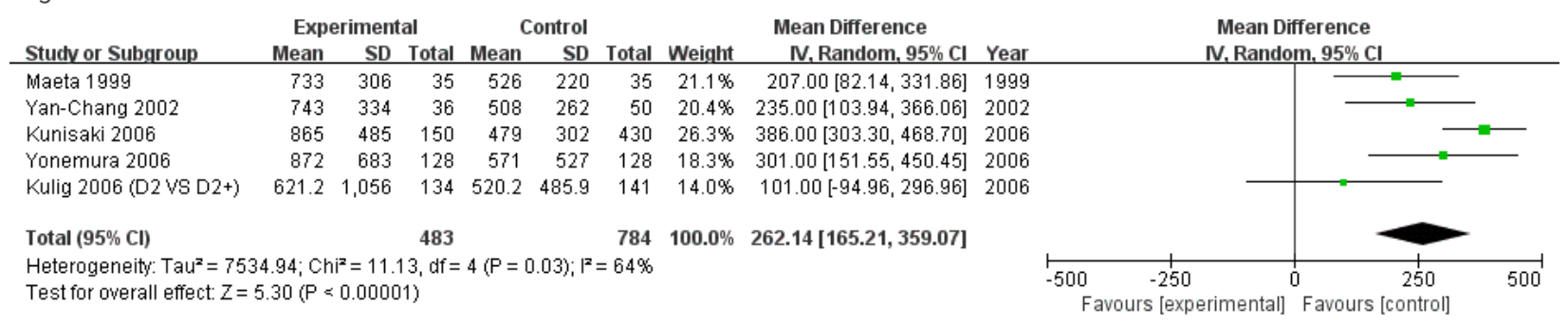


Figure 5

Legend not included with this version.

Experimental Control

Risk Ratio

Risk Ratio

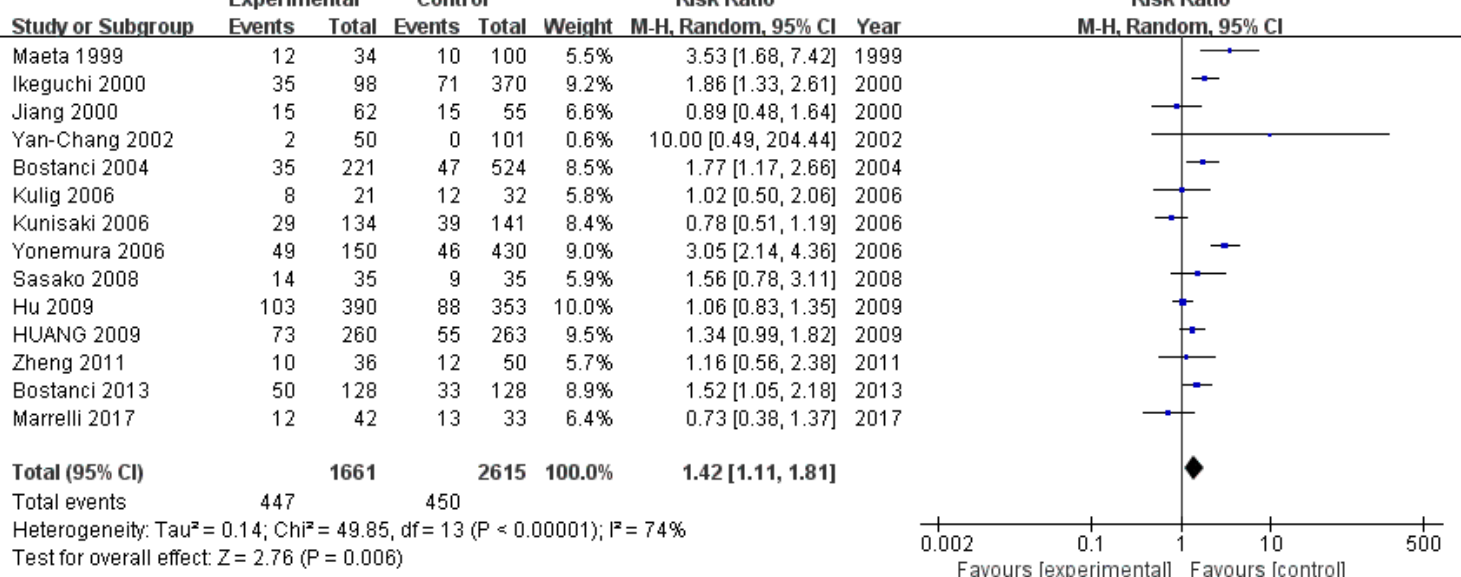

Figure 6

Legend not included with this version.

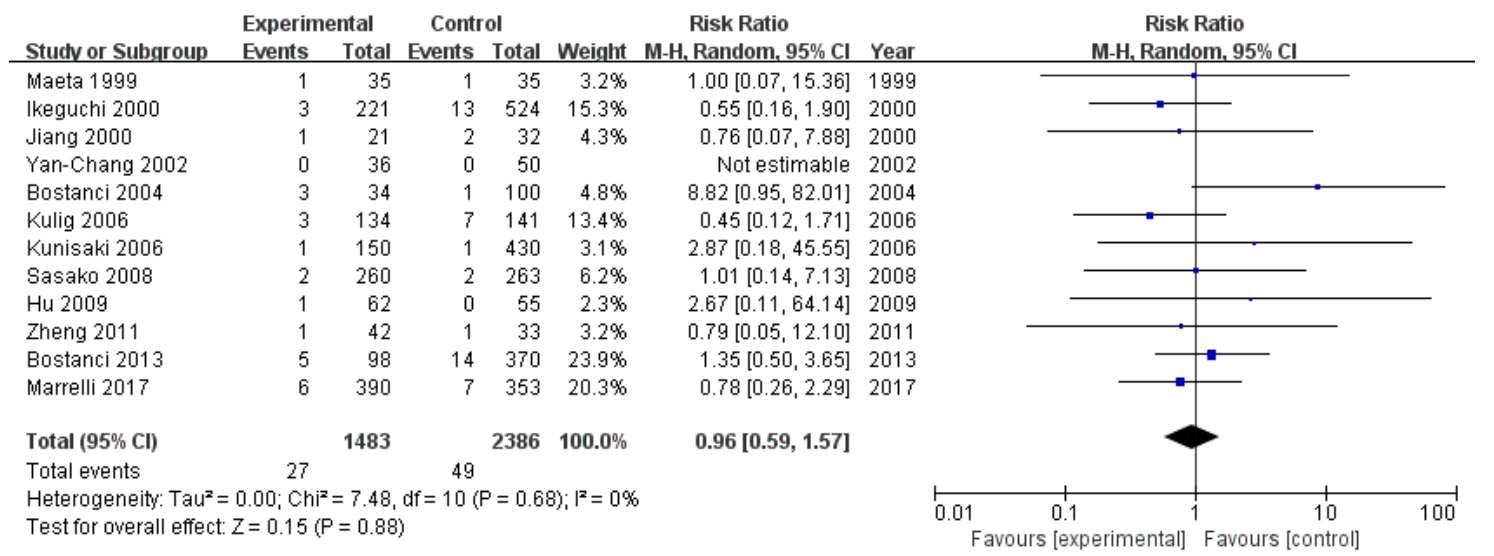

Figure 7

Legend not included with this version.

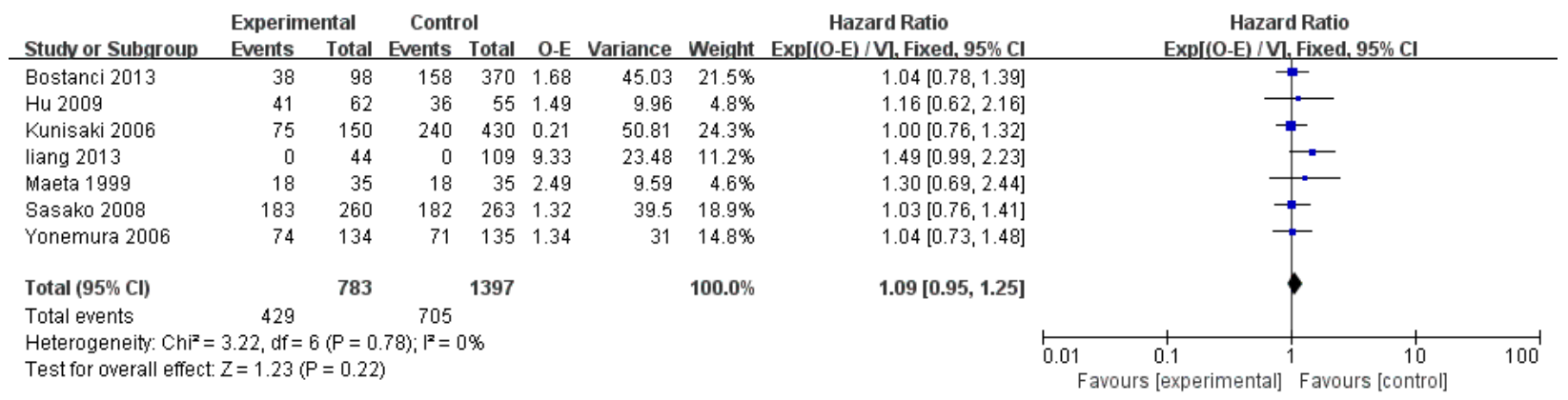

\section{Figure 8}

Legend not included with this version.

\section{Image not available with this version}


Image not available with this version.

\section{Supplementary Files}

This is a list of supplementary files associated with this preprint. Click to download.

- PRISMA2009Checklist.docx 